\title{
AN X-RAY ALL SKY MONITOR FOR A JAPANESE EXPERIMENTAL MODULE ON THE SPACE STATION
}

\author{
M. MATSUOKA, N. KAWAI, T. IMAI, M. YAMAUCHI, A. YOSHIDA, \\ T. KOHNO, A. YONEDA \\ The Institute of Physical and Chemical Research, \\ 2-1 Hirosawa, Wako, Saitama 351-01, Japan \\ and \\ H.TSUNEMI \\ Department of Physics, Faculty of Science, Osaka University, \\ 1-1 Machikaneyama-cho, Toyonaka, Osaka 560, Japan
}

\begin{abstract}
We propose an X-ray all sky monitor for Japanese Experimental Module (JEM) on the space station. Considering practical circumstances, we show as a case study that the all sky monitor with slit hole cameras is most promising for monitoring the short-term and long-term X-ray transients. We call this all sky monitor as MAXI (Monitor of Allsky X-ray Image). Position determination of gamma-ray bursts could be achieved with accuracy less than one degree observing the X-ray component of the burst. Weak X-ray sources such as active galactic nuclei could be also monitored with time resolution less than one day. The X-ray all sky monitor will work to discover X-ray novae and transient phenomena and give us the alarm for further detailed observations. The obtained data will be also used for archival study.
\end{abstract}

\section{Introduction}

Most celestial objects have potential to emit X-rays. X-ray luminosities from various objects are variable on timescales of wide range. Limited space and observation time on satellites disable us to monitor these variable $\mathrm{X}$-ray sources continuously.

Although X-ray all sky monitors have been performed by several satellites, duty cycles for observations are not enough for monitoring all the sky and also they have not yet monitored weak X-ray sources such as active galactic nuclei (Priedhorsky and Holt, 1987). It is, however, very promissing to achieve a comprehensive $\mathrm{X}$-ray all sky monitor on the space station (Matsuoka et al. 1988).

Space station program being promoted by NASA has just entered into its detailed design and development phases. As the first step of scientific payload to be attached to Japanese Experimental Module (JEM) we propose the MAXI (Monitor of All-sky X-ray Image) which is able to detect rapid variabilities from strong $\mathrm{X}$-ray sources as well as long-term variabilities from weak $\mathrm{X}$-ray sources. This paper gives an outline of the MAXI on the space station.

\section{The all sky monitor suitable for the space station}

We discuss on a practical X-ray all sky monitor suitable for the NASA space station as a scientific payload in the first phase. It is suggested that the MAXI (Monitor

Y. Kondo (ed.), Observatories in Earth Orbit and Beyond, 463-468.

(C) 1990 Kluwer Academic Publishers. Printed in The Netherlands. 


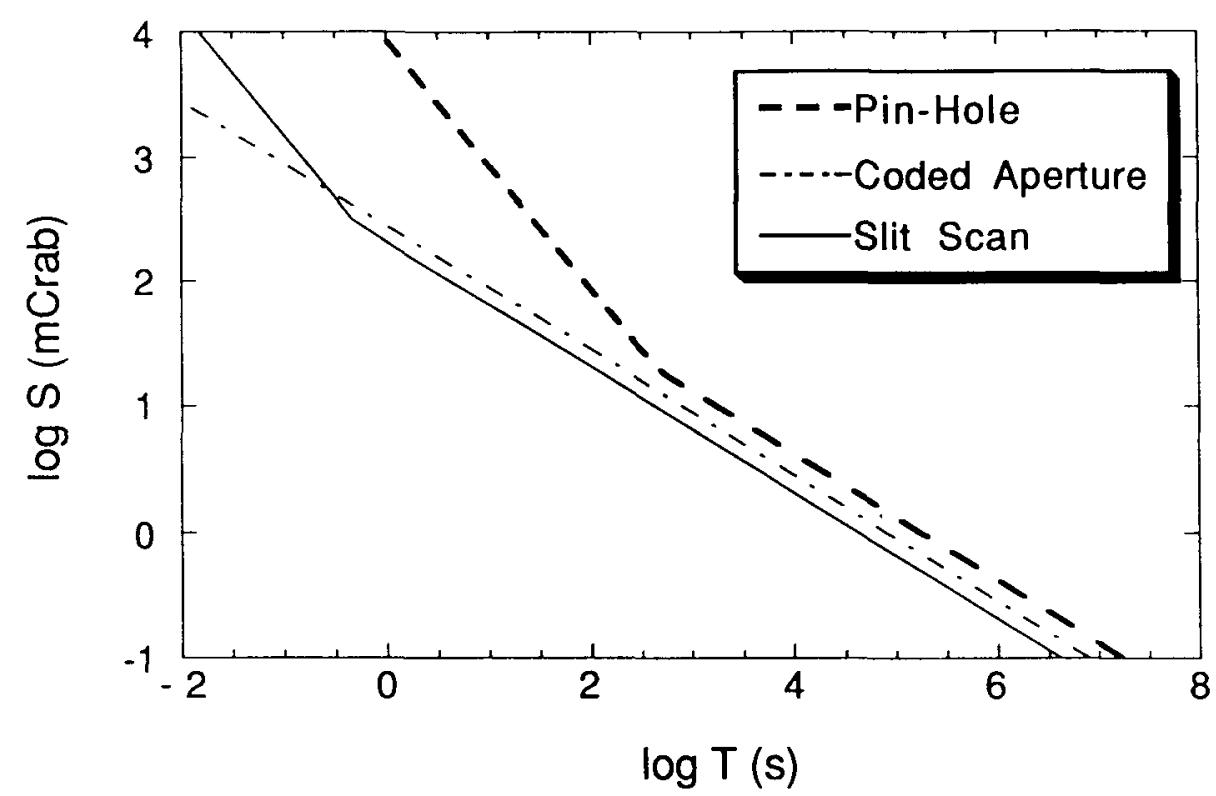

Fig. 1. The time vs. intensities to be required to detect a source with counts more than 5 photons as well as with a significant level of $5 \sigma$.

of All-sky X-ray Image) is attached to Japanese Experimental Module (JEM) on the Space Station. We will take into account the following problems to design the practical MAXI:

(1) Low cost and simple system for manufacturing, operation and maintenance.

(2) Moderate requirements for weight, electric power and data transmitting rate.

(3) Capability of partial achievement of scientific objectives by a single module.

(4) No requirement of an attitude control system.

(5) Providing of useful data for the community in astrophysics.

(6) Promotion of international collaboration for this payload.

(7) Desirable joint observations with wide band burst monitors such as optical/UV/IR and gamma-rays.

(8) Capability of optional instruments to grade up the data.

Here we supplement a few points for items (3) and (8). The item (3) means that the payload consists of several modules, but a single module is capable of partial achievement of scientific objectives independently. In item (8) astronauts can sometimes operate the optional instruments. This is a merit of the manned space station, but this requirement is not indispensible.

On the other hand the following requirements for the MAXI are considered in addition to the above problems:

(a) Detection limit of the order of 1 mCrab enables us to detect $\mathrm{X}$-rays from active galactic nuclei. 


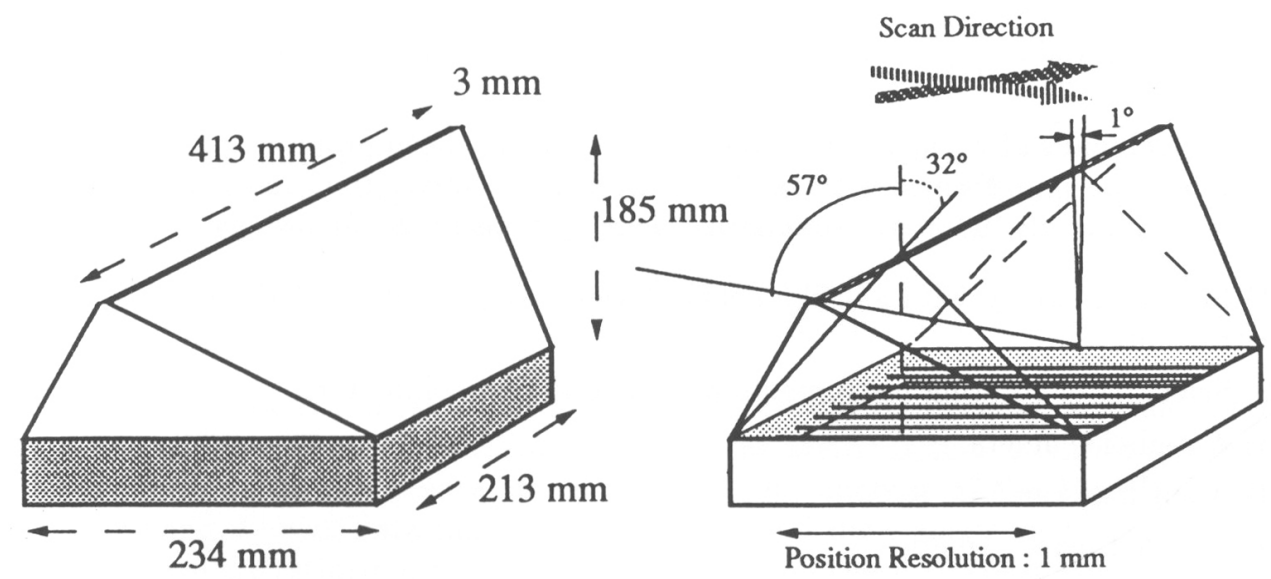

Fig. 2. A minimum unit of a pair of slit-hole cameras.

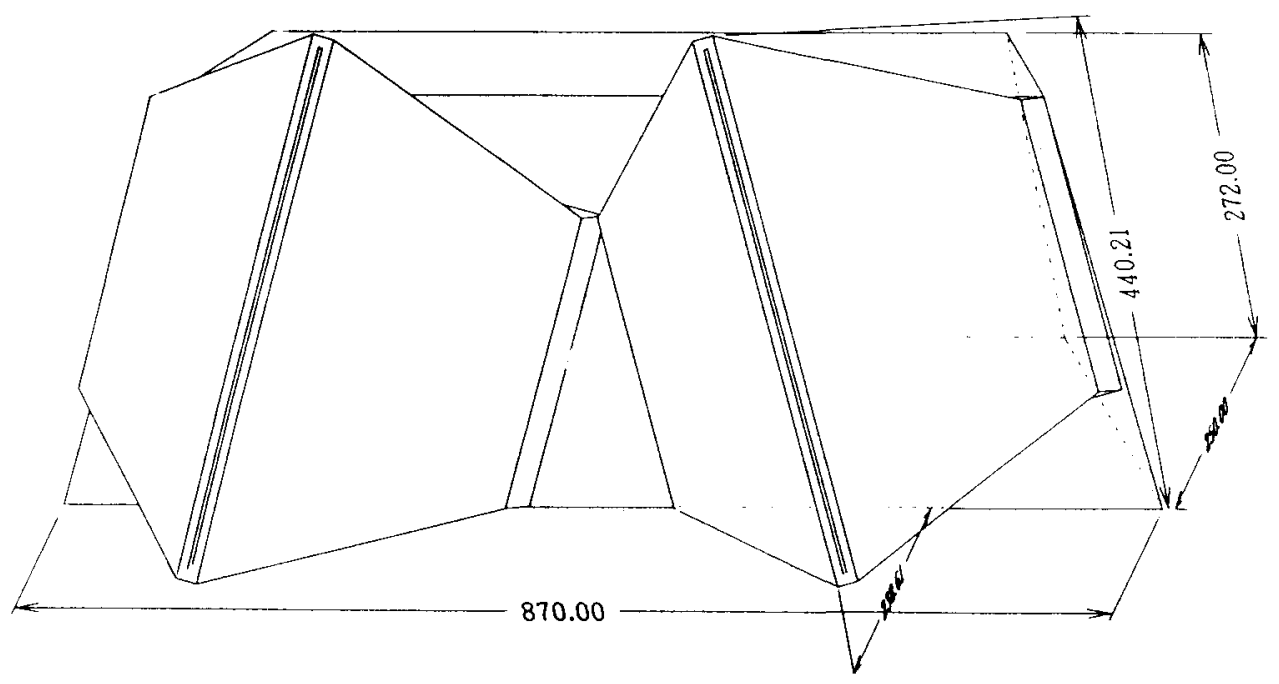

Fig. 3. An overview of a pair of cameras. 
(b) Spatial resolution is less than one degree to avoid confusion of sources with intensities stronger than $1 \mathrm{mCrab}$.

(c) A large duty cycle of observation time requires the X-ray image in a wide field of view.

Several kinds of X-ray all sky monitor systems have been proposed and most of them have been performed with satellites for each objective (Holt and Priedhorsky 1987; Caroli et al. 1987). We summarize these systems in the followings:

(1) Scanning survey with slit-like fields of view using rotation of the satellite or instrument.

(2) Scanning or rotating instruments with modulation collimators.

(3) A pinhole camera.

(4) A slit-hole camera.

(5) A coded mask camera.

For the space station we should employ the all sky monitor which has a duty cycle of $100 \%$ for intended field in the sky; that is, four systems above, (2), (3), (4) and (5).

The burst monitor of rotating modulation collimators on Hakucho have achieved fruitful discovery of new X-ray burst sources (Kondo et al. 1981). A modulation collimator on balloon gondola could determine a gamma-ray burst source in the hard X-ray band for the first time (Nishimura et al. 1978). The hard X-ray or soft gamma-ray all sky monitor using rotating modulation collimators is still being performed (Lund 1985). However, it has been pointed out mathematically that for a particular correlation map (or the corresponding data) X-ray intensity pattern is not uniquely determined even in the absence of statistical noise (Doi 1988). Therefore, the rotating modulation collimator is not suitable for imaging the all sky. Thus, in the following we will examine three systems of (3), (4) and (5) in detail.

We will compare three systems on the assumption that each system consists of 6 units of each effective area of $1000 \mathrm{~cm}^{2}$ and each covering field of one steradian; i.e., this system has total effective area of $6000 \mathrm{~cm}^{2}$, covering $2 \pi$ str. of sky. Non $\mathrm{X}$-ray background rate is assumed to be $6 \times 10^{-3} \mathrm{~s}^{-1} \mathrm{~cm}^{-2}$ for $2-10 \mathrm{keV}$. Position resolution is assumed to be $1^{\circ} \times 1^{\circ}$; i.e., the area of the pinhole camera and one pixel for the coded camera correspond to $0.29 \mathrm{~cm}^{2}$, while the area of the slit-hole camera is $17 \mathrm{~cm}^{2}$.

Fig. 1 shows the time which is necessary to detect a source with detection counts more than 5 photons as well as with a significant level of $5 \sigma$. This figure indicates that the slit-hole camera is most promising in respect of $\mathrm{S} / \mathrm{N}$ ratios over wide intensity range. A photon limit of this camera constrains a detection of bursts weaker than 1 Crab with a duration less than about 0.1 sec.

Although sources more than two would be often in the field of a slit pixel, each location could be determined using criss-cross method. Here, we can also use scanning information, because platforms on the JEM make one revolution every satellite orbit. 


\section{An outline of the MAXI}

The MAXI consists of 6 units, covering $2 \pi$ str. in total. One unit has a pair of cameras with two slit-holes, covering the field of one str. A pair of slit-holes are slanted each other by about 30 degrees. Data from a pair of cameras are similar to those in scanning mode from a pair of counters with slanting slat collimators. A large duty cycle of the sky by a slit-hole camera is an essential difference from a small duty cycle by a slat collimator system. A conceptional outline of a minimum unit of a pair of cameras is shown in Fig. 2, in which this detector is a one-dimentional proportional counter, having an effective area of $500 \mathrm{~cm}^{2}$. Fig. 3 shows an overview of a pair of counters.

Since locations of most bright X-ray sources are known precisely, we could determine their coordinates on the image map of the MAXI from the data themselves. Therefore, we do not need a star sensor for data analysis of the MAXI. On the other hand a lot of data must be processed. Total data points with resolution of one degree are estimated to be about $3 \times 10^{4}$ for all the sky except sky region near the sun. We estimate telemetry requirement to send the data. Telemetry data will be arranged with four modes; i.e., low, medium, and high bit rate modes and a burst mode. In the following we have considered that platforms on the JEM rotate one degree every about $15 \mathrm{sec}$.

Low bit rate mode: $1.15 \mathrm{k}$ bits/s for one energy band, time resolution of 7.5 sec and spatial data of 120 pixels. Medium bit rate mode: $7 \mathrm{k}$ bits/s for 4 energy bands, time resolution of $1 \mathrm{sec}$ and spatial data of 120 pixels. High bit rate mode: $32 \mathrm{k} \mathrm{bit} / \mathrm{s}$ for 64 energy channels, time resolution of $1 \mathrm{~m} \mathrm{sec}$ and spatial data of 240 pixels. Burst mode: total memory of about $100 \mathrm{k}$ bits for one burst data with duration of $10 \mathrm{sec}, 64$ energy channels and time resolution of about 1 msec. It would be possible to set a demountable mass storage memory such as optical disks for further information. In addition to the standard telemetry, more data can be taken if an optional mass storage system (MSS) is available. This MSS has to be operated by astronauts for change and retrieve of this memory once ten days or a few months. Optical disks or magnetic tapes could be available for the MSS.

The weight, size and power consumption of MAXI can be made to conform to the JEM specification. The one dimensional proportional counters should be manufactured carefully to keep the long-term operation. Detailed design can be modified flexibly if necessary. For example, we can transport each unit of the MAXI separately with space shuttles or Japanese HII rockets and build up one by one on the space station.

\section{References}

Caroli, E. et al.: 1987, Space Sci. Rev. 45, 349

Doi, K.: 1988, LxR Report "Intrinsic ambiguity in the X-ray image data obtained with the rotating modulation collimator system", No.8801

Holt, S.S. and Priedhorsky, W.: 1987, Space Sci. Rev. 45, 269

Kondo, I. et al.: 1981, Space Sci. Instr. 5, 211

Lund, N.: 1985, SPIE Proc. of X-ray Instrumentation in Astronomy, 597, 95 
Matsuoka, M., Kawai, N., and Tsunemi, H.: 1988, IPCR CR-13 prepared for a reference mission to be attached to the JEM on the space station,

Nishimura, J., et al.: 1978, Nature 272, 337

Priedhorsky, W.C. and Holt, S.S.: 1987, Space Sci. Rev. 45, 291 